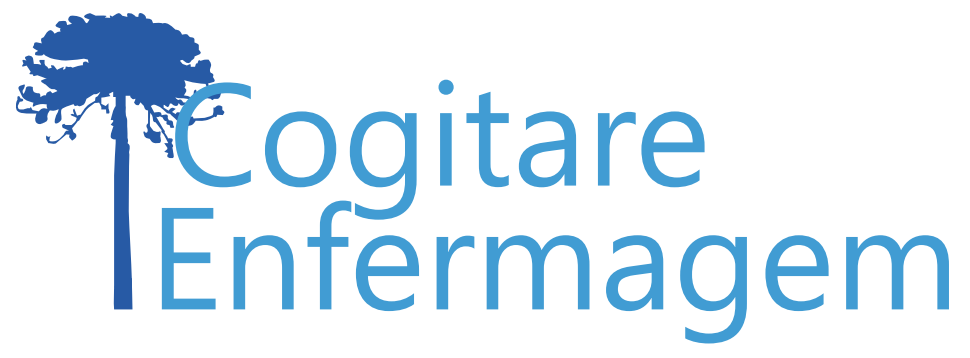

\title{
PLANEJAMENTO OPERACIONAL DURANTE A PANDEMIA DE COVID-19: COMPARAÇÃO ENTRE RECOMENDAÇÕES DA ORGANIZAÇÃO MUNDIAL DA SAÚDE E O PLANO DE CONTINGÊNCIA NACIONAL
}

Nila Larisse Silva de Albuquerque ${ }^{1}$

\begin{abstract}
RESUMO
Objetivo: frente à divulgação do guia COVID-19 - Operational Planning Guidelines to Support Country Preparedness and Response, pela Organização Mundial da Saúde, esta comunicação buscou comparar o conteúdo do Plano de Contingência Nacional às orientações da Organização Mundial da Saúde.

Desenvolvimento: Severe Acute Respiratory Syndrome Coronavirus 2 (SARS-Cov2), causador da COVID-19, apresenta transmissão comunitária sustentada no Brasil desde março de 2020. O país lançou seu Plano de Contingência Nacional para Infecção Humana pelo novo Coronavírus COVID-19, em fevereiro de 2020, no intuito de orientar a resposta nacional de combate à doença. Conclusão: o documento brasileiro atende parcialmente às recomendações do guia internacional e concentra suas principais lacunas nos pilares sobre pontos de entrada, prevenção e controle da infecção por SARS-CoV-2 COVID-19 em equipamentos de saúde e espaços comunitários, manejo de casos suspeitos e confirmados e suporte operacional e logístico.
\end{abstract}

DESCRITORES: Planejamento Operacional; Organização Mundial da Saúde; Pandemias.

COMO REFERENCIAR ESTE ARTIGO: Albuquerque NLS de. Planejamento operacional durante a pandemia de Covid-19: comparação entre recomendações da Organização Mundial da Saúde e o Plano de Contingência Nacional. Cogitare enferm. [Internet]. 2020 [acesso em "colocar data de acesso, dia, mês abreviado e ano"]; 25. Disponível em: http://dx.doi.org/10.5380/ce.v25i0.72659.

Este obra está licenciado com uma Licença Creative Commons Atribuição 4.0 Internacional. 


\title{
OPERATIONAL PLANNING DURING THE COVID-19 PANDEMIC: COMPARISON BETWEEN THE WHO RECOMMENDATIONS AND THE BRAZILIAN NATIONAL CONTINGENCY PLAN
}

\begin{abstract}
Objective: In view of the publication of the COVID-19 guide - Operational Planning Guidelines to Support Country Preparedness and Response, by the World Health Organization, this paper attempts to compare the content of the Brazilian National Contingency Plan to the WHO guidelines.

Development: Severe Acute Respiratory Syndrome Coronavirus 2 (SARS-Cov2), the virus that causes COVID-19, has a sustained community transmission in Brazil since March 2020. The country launched the National Contingency Plan for Human Infection with the new COVID-19 Coronavirus in February 2020, in order to guide the national response to fight the disease. Conclusion: The Brazilian document only partially meets the recommendations of the international guide, the main gaps being the points of entry, prevention, and control of the infection caused by SARS-CoV-2 in health equipment and community spaces, handling suspected and confirmed cases, and operational and logistical support.
\end{abstract}

DESCRIPTORS: Operational Planning; World Health Organization; Pandemics.

\section{PLANIFICACIÓN OPERATIVA DURANTE LA PANDEMIA DE COVID-19: COMPARACIOON ENTRE LAS RECOMENDACIONES DE LA ORGANIZACIÓN MUNDIAL DE LA SALUD Y EL PLAN DE CONTINGENCIA NACIONAL}

\begin{abstract}
RESUMEN:
Objetivo: en vista de la divulgación de la guía COVID-19 - Operational Planning Guidelines to Support Country Preparedness and Response, por parte de la Organización Mundial de la Salud, este comunicado tuvo como objetivo comparar el contenido del Plan de Contingencia nacional con las pautas de la Organización Mundial de la Salud.

Desarrollo: Severe Acute Respiratory Syndrome Coronavirus 2 (SARS-Cov2), que causa el COVID-19, presenta transmisión comunitaria en Brasil desde marzo de 2020. El país lanzó su Plan de Contingencia Nacional para la Infección Humana por el nuevo Coronavirus COVID-19 en febrero de 2020, con la intención de orientar la respuesta nacional de lucha contra la enfermedad.

Conclusión: el documento brasileño cumple parcialmente las recomendaciones de la guía internacional y concentra sus principales déficits en los pilares sobre puntos de entrada, prevención y control de la infección SARS-CoV-2 COVID-19 en equipamientos de salud y espacios comunitarios, manejo de casos sospechados y confirmados, y soporte operativo y logístico.
\end{abstract}

DESCRIPTORES: Planificación operativa; Organización Mundial de la Salud; Pandemias. 
COVID-19 é uma doença infecciosa causada pelo vírus Severe Acute Respiratory Syndrome Coronavirus 2 (SARS-CoV-2), convencionalmente chamado de novo coronavírus. Possui período médio de incubação de 5,5 dias e aparecimento de sintomas em até 11 dias em $97,5 \%$ das pessoas infectadas ${ }^{(1)}$.

Ainda que as informações epidemiológicas sobre a COVID-19 sejam limitadas, evidências apontam que a idade média dos pacientes admitidos em unidades hospitalares pela doença é de 49 anos e os sintomas mais comuns são: febre (98\%), tosse (76\%), dispneia $(55 \%)$ e fadiga muscular $(44 \%)^{(2)}$. Pacientes com comorbidades pré-existentes à infecção desenvolvem formas mais severas da doença e possuem taxas de mortalidade significativamente elevadas ${ }^{(3)}$.

O avanço sustentado de casos de COVID-19 no mundo tem sido acompanhado da formulação de planos para respostas rápidas à disseminação da doença ${ }^{(4)}$. Nesses, estão incluídas ações de detecção precoce, isolamento, vigilância epidemiológica, medidas de prevenção e controle, bem como a avaliação de impactos sanitários ${ }^{(3)}$.

Com vistas a fornecer direcionamentos práticos para gestores e planejadores de políticas de saúde desenvolverem planos estratégicos de preparação e resposta para o combate à COVID-19, a Organização Mundial da Saúde (OMS) divulgou o documento "COVID-19: Operational Planning Guidelines to Support Country Preparedness and Response" (5).

Por sua vez, a Secretaria de Vigilância em Saúde do Ministério da Saúde do Brasil lançou o Plano de Contingência Nacional para Infecção Humana pelo novo Coronavírus COVID-19(6), o qual é composto por três níveis de resposta: Alerta, Perigo Iminente e Emergência em Saúde Pública.

Desde meados do mês de março de 2020, o Brasil se encontra no terceiro nível de resposta, o qual corresponde à situação em que há confirmação de transmissão local do COVID-19 em território nacional. Esse cenário demanda planejamento e implementação das melhores práticas recomendadas ao combate à disseminação da infecção. Com vistas a fomentar reflexões sobre a otimização do planejamento da resposta brasileira à pandemia, buscou-se nesta comunicação comparar o conteúdo do Plano de Contingência Nacional(6) com as diretrizes publicadas pela OMS em seu guia de elaboração de planos estratégicos de preparação e resposta para o combate ao COVID-19(4).

\section{DESENVOLVIMENTO}

O Plano de Contingência Nacional(6) determina a organização do nível de emergência de saúde pública de importância nacional em duas fases: fase de contenção, na qual se busca evitar a transmissão sustentada do vírus, e fase de mitigação, que tem início a partir do registro de 100 casos positivos do novo coronavírus. No Brasil, a fase de mitigação foi alcançada em cerca de 15 dias após a confirmação do primeiro caso de COVID-19 no país. Na Itália, o intervalo de tempo para alcançar o marco de 100 casos foi de aproximadamente 20 dias e nos Estados Unidos, sete dias ${ }^{(7)^{3}}$.

Nessa fase, o nível de resposta à emergência de saúde pública de importância nacional é acionado e o Plano de Contingência Nacional(6), que estabelece as seguintes medidas de resposta: vigilância, suporte laboratorial, medidas de controle de infecção, assistência, assistência farmacêutica, vigilância sanitária, comunicação de risco e gestão(6).

Essas prioridades vão ao encontro do documento COVID-19: Operational Planning Guidelines to Support Country Preparedness and Response ${ }^{(4)}$. Entretanto, há lacunas e 
divergências entre o Plano de Contingência Nacional(6) e as recomendações da Organização Mundial da Saúde ${ }^{(5)}$, que serão apresentadas a seguir, no intuito de discutir e fomentar as respostas brasileiras ao cenário atual de pandemia.

\section{Pilares de ação}

O primeiro pilar apresentado no documento da $\mathrm{OMS}^{(5)}$ refere-se às ações nacionais de planejamento e monitoramento. Recomenda-se que os planos nacionais de contingência apresentem mecanismos de resposta à situação com o envolvimento de ministérios relevantes e parceiros-chave da sociedade, no intuito de prover manejo coordenado dos casos de COVID-19.

O Plano de Contingência Nacional(6) explicita a estrutura de comando do Centro de Operações de Emergências para resposta ao novo coronavírus e dos sub-comitês técnicos para suporte à tomada de decisão. Entretanto, não contempla algumas ações recomendadas pela OMS nesse âmbito, tais como estimação de recursos necessários para conter as ocorrências de COVID-19 no país e articulação de estratégias multisetoriais para prover aportes financeiros necessários.

Tem-se como segundo pilar a comunicação sobre riscos e o engajamento da comunidade, no qual sugere-se que sejam disponibilizadas à população as evidências disponíveis sobre o COVID-19 e descritas as ações que estão sendo realizadas para lidar com a pandemia. O tópico sobre comunicação de risco no plano de contingência brasileiro ${ }^{(6)}$ assegura a realização dessas ações, incluindo a disseminação de informaçõ̃es acuradas e o esclarecimento de rumores em canais adequados para comunicação.

Ressalta-se que universidades possuem papel preponderante na atenção às demais recomendações da OMS nesse âmbito, tais como: condução de avaliações de comportamento para identificar canais preferíveis de comunicação com a comunidade; preparo de mensagens a serem enviadas em nível local, incluindo aos grupos mais vulneráveis; identificação de grupos comunitários e redes locais; e disseminação de materiais educativos em locais e canais estratégicos.

A OMS destaca que, em países com transmissão local como o Brasil, o terceiro pilar, relativo à vigilância, formação de times de resposta rápida e investigação de casos, possui papel indispensável no combate eficaz à pandemia. As ações devem ser pautadas na adoção de protocolos para rastreamento de contatos e monitoramento de casos confirmados. No entanto, o plano nacional não informa quais protocolos serão adotados para rastrear contatos e monitorar casos confirmados. Relatos advindos da Coreia do Sul(8) e Hong Kong (9) informam que, nesses países, tais procedimentos foram realizados por GPS, ligações telefônicas e mensagens de texto.

Ainda no âmbito da vigilância, a OMS recomenda que as tendências da doença sejam reportadas, incluindo dados clínicos, taxa de mortalidade e ocorrência em grupos de risco ${ }^{(5)}$. Já o plano de contingência nacional|(6) menciona a utilização de Boletins Epidemiológicos para divulgação das informações sobre a situação dos casos no Brasil. Sugere-se que os dados epidemiológicos sejam disponibilizados na íntegra em plataformas de acesso aberto, respeitando o anonimato dos pacientes, no intuito de prover fontes de informação para a realização de estudos nacionais sobre a COVID-19. Novamente as universidades possuem papel de destaque, ao oportunamente alocar recursos humanos para o desenvolvimento de análises epidemiológicas e modelos preditivos a partir dos dados disponibilizados.

O quarto pilar trata dos pontos de entrada de casos de COVID-19: aeroportos, portos e passagens em fronteiras. O Plano de Contingência Nacional ${ }^{(6)}$ contempla as recomendações da $\mathrm{OMS}^{(5)}$ para essas circunstâncias, com exceção à orientação sobre a preparação de estruturas para isolamento rápido de passageiros sintomáticos em pontos de entrada. Discussões sobre esse ponto, bem como eventuais fechamentos de fronteiras, poderão fazer parte da reavaliação contínua do documento. 
O quinto pilar consiste na estrutura de laboratórios nacionais para atender à demanda crescente por testagem para detecção de infecção por SARS-CoV-2. Segundo o guia da OMS, todos os países devem preparar laboratórios nacionais para lidar com testagem em larga escala para COVID-19(6). Ressalta-se que a capacidade de testagem no Brasil se concentra nos Laboratórios Centrais de Saúde Pública (LACEN) e poderá ser expandida com a colaboração de laboratórios de universidades e da rede privada, desde que atendam às regulamentações sanitárias pertinentes.

O sexto pilar do guia internacional(5) trata da prevenção e controle da infecção por COVID-19 em equipamentos de saúde e espaços comunitários. Sobre o assunto, o plano de contingência nacional(6) tem por foco prover orientações aos serviços de saúde quanto à prevenção e o controle de infecção para casos suspeitos e confirmados de COVID-19.

Entretanto, a OMS determina ações adicionais nesse âmbito, tais como: avaliação da capacidade de prevenir e controlar infecções por SARS-CoV-2 em sistemas públicos e privados de saúde, bem como farmácias e espaços comunitários; e orientação de práticas de controle e prevenção em escolas, supermercados, transporte público e domicílios ${ }^{(5)}$. Assim, considera-se que posteriores atualizações do plano nacional possam adicionar e reforçar orientações sobre prevenção e controle da infecção, com base em procedimentos já consensualmente aceitos na literatura ${ }^{(10)}$ e voltadas não somente a equipamentos de saúde, mas também a domicílios e comunidades.

Há lacunas no plano nacional(6) no que tange ao delineamento de estratégias de prevenção e controle de infecções que levam à COVID-19 entre profissionais de saúde. Apesar de a OMS recomendar que os planos informem como será realizado registro e investigação de todos os casos de infecção em profissionais de saúde e a implementação de mecanismos de triagem, detecção precoce e controle de fontes de infecção nos espaços de atuação das equipes de saúde ${ }^{(5)}$, esses pontos não são abordados no direcionamento brasileiro.

Recomenda-se que outros segmentos da sociedade, como grupos de pesquisa e extensão das universidades e conselhos de classe, contribuam com o delineamento de estratégias nesse âmbito, principalmente no que concerne à organização de espaços de triagem voltados aos profissionais de saúde em linha de frente na assistência.

Estratégias de manejo em equipamentos de saúde devem contemplar o aumento exponencial de casos suspeitos e confirmados para COVID-19, e esse é o sétimo pilar do guia da $\mathrm{OMS}^{(5)}$. O preparo para a condução adequada de casos envolve mapeamento de populações vulneráveis e equipamentos públicos e privados de saúde capazes de receber casos suspeitos de COVID-19, bem como identificação de locais alternativos que possam ser utilizados para prover tratamento, avaliação da capacidade de leitos de terapia intensiva, e planejamento de orientações de autocuidado a serem fornecidas àqueles com sintomas leves da COVID-19.

No Plano Nacional de Contingência ${ }^{(6)}$, o funcionamento dessas ações de preparação no país é pouco explorado, no entanto, são apresentadas diretrizes voltadas ao funcionamento adequado e à ampliação das redes de atenção à saúde diante do aumento de casos de COVID-19.

Ainda no pilar sobre manejo de casos, a OMS recomenda que os planos de resposta nacionais à COVID-19 descrevam ações para avaliar a viabilidade de inserir métodos diagnósticos, terapêuticas e vacinas em testes clínicos ${ }^{(5)}$. Em virtude da pandemia, novos testes diagnósticos foram adotados internacionalmente e, no Brasil, mediante autorização da Agência Nacional de Vigilância Sanitária.

Ressalta-se que, apesar de o plano nacional mencionar somente o método diagnóstico de transcrição reversa da reação em cadeia da polimerase (do inglês Reverse Transcription Polymerase Chain Reaction - RT-PCR) em tempo real(6), a adoção de novos meios diagnósticos demanda ações específicas de vigilância sanitária. Recomendações semelhantes são aplicadas à realização de estudos especiais para testar uso por compaixão 
de drogas já existentes, uso off label e desenvolvimento de novas terapêuticas.

Por fim, o guia da OMS apresenta seu oitavo e último pilar: suporte operacional e logístico. Sabe-se que, diante da pandemia de COVID-19 e seus impactos em serviços de saúde, arranjos logísticos devem ser reforçados. Para tanto, a OMS recomenda que sejam mapeados recursos e insumos disponíveis no setor local e nacional de saúde, e que o sistema de controle e gerenciamento da cadeia de suprimentos médico-hospitalares, bem como os processos de compra, sejam redirecionados para atender à demanda ${ }^{(5)}$.

O Plano de Contingência Nacional(6) propõe garantir e monitorar estoque estratégico de insumos laboratoriais para diagnóstico e atendimento de casos suspeitos e confirmados. No entanto, não especifica como a cadeia de produção, compra e distribuição de insumos será readequada para atender ao contexto. No cenário atual, em que países com disseminação em larga escala de COVID-19 enfrentam baixa disponibilidade de insumos e equipamentos de proteção individual, delinear o funcionamento do suporte operacional e logístico é indispensável para manter a prestação de serviços de resposta à pandemia pelo COVID-19.

Conforme a OMS, também deve fazer parte do plano de contingência a manutenção de serviços essenciais, tais como saneamento básico e tratamento da água, provimento de energia, produção alimentícia e telecomunicações ${ }^{(5)}$. O Plano de Contingência Nacional não contempla esse aspecto, no entanto, o Decreto $n^{\circ} 10.282^{(11)}$ define os serviços e atividades essenciais que não poderão ser interrompidos durante a vigência do estado de calamidade no Brasil.

\section{CONCLUSÃO}

Em suma, o Plano de Contingência Nacional para Infecção Humana pelo novo Coronavírus COVID-19(6) atende parcialmente às recomendações do guia publicado pela $\mathrm{OMS}^{(5)}$. As principais lacunas concentram-se nos pilares sobre pontos de entrada, prevenção e controle da infecção por SARS-CoV2, que leva à COVID-19, em equipamentos de saúde e espaços comunitários, manejo de casos e suporte operacional e logístico. Considera-se que o detalhamento de estratégias voltadas a esses pilares é indispensável ao aprimoramento da resposta à pandemia por COVID-19 no Brasil.

\section{REFERÊNCIAS}

1. Lauer SA, Grantz KH, Bi Q, Jones FK, Zheng $\mathrm{Q}$, Meredith HR, et al. The incubation period of Coronavirus disease 2019 (COVID-19) from publicly reported confirmed cases: estimation and application. Ann Intern Med. [Internet]. 2020 [acesso em 02 abr 2020]. Disponível em: https://doi.org/10.7326/M20$\underline{0504 .}$

2. Huang C, Wang Y, Li X, Ren L, Zhao J, Hu Y, et al. Clinical features of patients infected with 2019 novel coronavirus in Wuhan, China. Lancet. [Internet]. 2020 [acesso em 26 mar 2020]; 395(10223). Disponível em: https://doi.org/10.1016/S0140-6736(20)30183-5.

3. Li S, Wang Y, Xue J, Zhao N, Zhu T. The impact of COVID-19 epidemic declaration on psychological consequences: a study on active weibo users. Intl J Environ Res Pub Health [Internet]. 2020 [acesso em 26 mar 2020]; 17(6). Disponível em: https://doi.org/10.3390/ijerph17062032.

4. Lake MA. What we know so far: COVID-19 current clinical knowledge and research. Clin Med (Lond) [Internet]. 2020 [acesso em 27 mar 2020]; 20(2). Disponível em: https://doi.org/10.7861/clinmed.2019coron. 
5. World Health Organization (WHO). COVID-19 Strategic preparedness and response plan operational planning guidelines to support country preparedness and response. Geneva: WHO; 2020.

6. Ministério da Saúde (BR). Secretaria de Vigilância em Saúde. Plano de Contingência Nacional para Infecção Humana pelo novo Coronavírus COVID-19. [Internet] Brasília: Ministério da Saúde; 2020 [acesso em 19 mar 2020]. Disponível em: https://portalarquivos2.saude.gov.br/images/pdf/2020/fevereiro/13/ plano-contingencia-coronavirus-COVID19.pdf.

7. WorldoMeter. COVID-19 Coronavirus Pandemic. [Internet]. 2020 [acesso em 24 mar 2020]. Disponível em: https://www.worldometers.info/coronavirus/.

8. Kim HJ. South Korea learned its successful Covid-19 strategy from a previous coronavirus outbreak: MERS. Bull At Sci. [Internet]. 2020 [acesso em 26 mar 2020]. Disponível em: https://thebulletin. org/2020/03/south-korea-learned-its-successful-covid-19-strategy-from-a-previous-coronavirus-outbreakmers/\#.

9. Privacy International. Hong Kong: tracker wristbands help enforce quarantine. [Internet]. 2020 [acesso em 26 mar 2020]. Disponível em: https://privacyinternational.org/examples/3477/hong-kong-trackerwristbands-help-enforce-quarantine.

10. Agarwal A, Nagi N, Chatterjee P, Sarkar S, Mourya D, Sahay RR, et al. Guidance for building a dedicated health facility to contain the spread of the 2019 novel coronavirus outbreak. Indian J Med Res [Internet]. 2020 [acesso em 30 mar 2020]. Disponível em: https://doi.org/10.4103/ijmr.IJMR 51820.

11. Ministério da Saúde (BR). Decreto n. 10.282, de 20 de março de 2020: regulamenta a Lei n. 13.979, de 6 de fevereiro de 2020, para definir os serviços públicos e as atividades essenciais. Diário Oficial da União, [Internet]. 2020 [acesso em 30 mar 2020]. Disponível em: http://www.planalto.gov.br/ccivil_03/ ato2019-2022/2020/decreto/D10282.htm.

Recebido: 03/04/2020

Finalizado: $17 / 04 / 2020$

Editora associada: Luciana Puchalski Kalinke

Autor Correspondente:

Nila Larisse Silva de Albuquerque

Universidade Federal do Ceará

R. Alexandre Baraúna, 1115 - 60430-160 - Fortaleza, CE, Brasil

E-mail: larisseufc@hotmail.com

Contribuição dos autores:

Contribuições substanciais para a concepção ou desenho do estudo; ou a aquisição, análise ou interpretação de dados do estudo - NLSA

Elaboração e revisão crítica do conteúdo intelectual do estudo - NLSA

Aprovação da versão final do estudo a ser publicado - NLSA

Responsável por todos os aspectos do estudo, assegurando as questões de precisão ou integridade de qualquer parte do estudo - NLSA 